\title{
PHOTOGRAMMETRY AND VIDEOGRAMMETRY METHODS FOR SOLAR SAILS AND OTHER GOSSAMER STRUCTURES
}

\author{
Jonathan T. Black, George Washington University, NASA Langley Research Center \\ Richard S. Pappa, NASA Langley Research Center
}

\begin{abstract}
$\underline{\text { Abstract }}$
Ultra-lightweight and inflatable gossamer space structures are designed to be tightly packaged for launch, then deploy or inflate once in space. These properties will allow for in-space construction of very large structures 10 to 1000 meters in size such as solar sails, inflatable antennae, and space solar power stations using a single launch. Solar sails are of particular interest because of their potential for propellantless propulsion. Gossamer structures do, however, have significant complications. Their low mass and high flexibility make them very difficult to test on the ground. The added mass and stiffness of attached measurement devices can significantly alter the static and dynamic properties of the structure. This complication necessitates an alternative approach for characterization. This paper discusses the development and application of photogrammetry and videogrammetry methods for the static and dynamic characterization of gossamer structures, as four specific solar sail applications demonstrate. The applications prove that high-resolution, full-field, non-contact static measurements of solar sails using dot projection photogrammetry are possible as well as full-field, noncontact, dynamic characterization using dot projection videogrammetry.
\end{abstract}

\section{$\underline{\text { Introduction }}$}

The term gossamer is generally applied to ultra-low-mass space structures. Frequently these structures are designed to be tightly packaged for launch, and then to deploy or inflate once in space. Most gossamer space structures rely on ultra-thin membranes and inflatable tubes to achieve a reduction, compared to standard space hardware, in launch mass by a factor of 10 and in launch volume by a factor of 50. The technology has been adapted for possible use in a wide variety of applications, including deployable ballutes for aerobraking on Mars, telescope sunshields, and membrane space solar arrays. [1 - 4]

Solar sails are studied because of their unique potential for providing propellantless propulsion. Through the momentum transfer of reflected photons of sunlight (sail membranes have highly reflective surfaces), solar sails can generate a small but continuous acceleration on the order of $1.0 \mathrm{~mm} / \mathrm{s}^{2}$. This constant thrust allows travel in non-Keplerian orbits enabling smaller versions less than 100 meters on a side (square sail) to hold an approximately stationary location relative to the Sun or the Earth, such as a polar observing satellite. Larger sails, over 150 meters per side, will be able to reach Jupiter in only two years and Pluto in just a decade [3-7].

Useful levels of continuous acceleration are only achievable in solar sails with very low areal mass densities. The pressure provided by sunlight is just $9.12 \times 10^{-6} \mathrm{~N} / \mathrm{m}^{2}$ at one $\mathrm{AU}$, meaning that the spacecraft must be very large and very lightweight - less than approximately 20 grams per square meter overall, including payload - to generate acceptable acceleration. Suitable membranes are less than $5 \mu \mathrm{m}$ thick with areal densities less than $7 \mathrm{~g} / \mathrm{m}^{2}[4,6,7]$. For comparison, standard $8.5 \times 11$ inch white paper is $100 \mu \mathrm{m}$ thick with a density of $75 \mathrm{~g} / \mathrm{m}^{2}$. While static and dynamic characterization of structures normally requires the attachment of accelerometers or other measurement devices, the added mass and stiffness of potentially hundreds of these devices could drastically alter the properties of the membrane structure. Therefore, a totally non-contact measurement method such as dot projection photogrammetry is preferred. This paper details the development of dot projection photogrammetry and videogrammetry methods for static and dynamic characterization of solar sail structures.

\section{Photogrammetry}

Photogrammetry is the science of making precise shape measurements from photographs. To generate these measurements, multiple images are loaded into the photogrammetry software and associated with the appropriate calibration parameters. These parameters are calculated independently of the measurement and allow the software to remove distortions in the images caused by lens curvature and imperfections, focus, aperture setting, and other camera factors and aberrations.

Totally non-contact photogrammetry uses a grid of targets created by a projector on the test article. Multiple digital cameras then image these targets. After the images are loaded into the photogrammetry software and assigned the calibration parameters, each of the projected targets are marked to sub-pixel accuracy using an automatic least squares matching (LSM) algorithm. The points, corresponding to the

$-1-$ 
exact centers of the targets, are then matched across the images and a bundle adjustment algorithm is run that simultaneously iterates on the camera and point locations, both in three-dimensional space. The final result of the process is a set of three-dimensional points called a "point cloud" that is exported for measurement $[7-11]$.

\section{Static Application Examples}

All three of the applications discussed in this section were conducted for dual purposes: to develop measurement methods suitable for use on actual solar sail spacecraft and to obtain measurements for validation of structural analytical models. The experiments used low-fidelity, generic solar sail test articles currently at NASA Langley Research Center. These low-fidelity test articles are sub-scale models manufactured from solar sail quality materials but are not directly scalable to full size solar sails. The developed methods will be applied to other current and future high-fidelity gossamer test structures and space missions.

\section{Low Density Full-field Two Meter Kapton Solar Sail}

This experiment was designed to assess the feasibility of using projected circular targets instead of attached retro-reflective targets on highly reflective membrane surfaces. The two meter per side, square, aluminized Kapton solar sail test article shown in Figure 1 was selected for static shape measurement because of its continuity (other test articles are divided into four quadrants). A pattern of approximately 400 dots was projected onto the membrane and imaged by four consumer digital cameras. The cameras were positioned at approximately $90^{\circ}$ separation at the corners of the sail and simultaneously photographed it to avoid possible membrane movement between images, as shown in Figure 2.

Figure 3 shows an actual image used in the photogrammetric processing, with the dot pattern clearly visible on the surface of the sail. The image is underexposed to create high-contrast targets optimal for photogrammetric measurement.

Several trouble spots are evident in the image in Figure 3. The long exposure time (30 seconds) required to obtain the images due to the reflective nature of the membrane allowed ambient light to also image on the sail, causing the background to appear much brighter than desired. In the image in Figure 3, ambient light imaging on the upper right corner of the sail drastically reduced the contrast between the dots and the membrane in that area, leading to a loss of target information. Also, there are several "hot spots" in areas where the sail curled, reflecting much more light into the camera than in the rest of image and washing out all data (i.e. targets) in that particular region. Two hot spots are visible in both bottom corners of the image in Figure 3. The low density of the dot pattern creates a low resolution measurement condition, meaning only the overall shape and not the intricate wrinkle pattern seen in Figure 1 can be adequately characterized from these images. While the long exposure time, hot spots, and low resolution do not preclude measurement, they detract from the achievable accuracy and precision of the process.

Figure 2 shows an image of the point cloud and camera locations created from the 3-D Viewer in the photogrammetry software, which can be studied to verify that the project processed correctly and that the setup and result are as expected. The four camera stations (one in each corner of the sail) and the final point cloud are positioned as expected. This result demonstrates that the camera positions have been calculated successfully and that the point location calculations have produced a nearly planer threedimensional cloud, expected when measuring a predominantly flat membrane.

Figure 4 demonstrates how the imperfections in the image in Figure 3 affect the photogrammetric processing of the project. It shows a close up view of the upper right corner of the sail in image in Figure 3 after the processing was completed. As discussed earlier, the ambient light in the room led to a loss of data in this region of the membrane. Some targets in the area are not visible and therefore could not be marked (marked targets are identified by an asterisk). Several other targets do not have sufficient contrast to be marked using the automatic LSM process and therefore were marked by hand, reducing precision. The greatest amount of wrinkling occurs at the corners of the membrane making them important in the measurement. The ambient light, therefore, has caused a loss of accuracy and data in one of the most critical areas of the sail.

The photogrammetry software estimates precision using error propagation techniques. The average of all the precision values for all of the points in the project was calculated to be $0.16 \mathrm{~mm}$, meaning that overall for the two meter sail, the measurements were precise to one part in $12,500(1: 12,500)$.

The three-dimensional point cloud corresponding to centers (centriods) of the targets was imported into a contour mapping program, which was used to visualize the membrane shape. The first step in the visualization involved the creation of a continuous surface from the imported, discrete data; a process called gridding. Several different algorithms are available to the user during grid creation, depending on the amount of smoothing and interpolation desired. Subsequently the grid was displayed as a shaded surface representing the out of plane deflection of the membrane surface. 
The results of the mapping for the gathered photogrammetry data are shown in Figure 5. The shaded surface shows the overall low-resolution shape of the membrane. This type of surface measurement is useful for assessing the overall shape of the structure (e.g. is it warped or flat), but is of little value for characterizing wrinkle patterns, which requires highresolution data. However this experiment does demonstrate the feasibility of using non-contact dot projection photogrammetry to obtain surface shape models of highly reflective aluminized membranes. A test to measure wrinkle pattern and amplitudes of this same test article is detailed in the following section.

\section{Wrinkle Test of Two Meter Kapton Solar Sail}

The wrinkle test described here was performed to investigate if dot projection photogrammetry could successfully measure small amplitude surface features on highly reflective solar sail membranes. Such data are important for validation of structural models and will lead to better prediction of the contribution of wrinkling to loss in propulsive efficiency, to predicting and preventing localized hot spots, to understanding membrane dynamics, and to providing an indication of the corresponding film stress. The two meter aluminized Kapton solar sail test article used for the previously described measurements (Figure 1) was selected. The wrinkle pattern of the membrane shown in Figure 1 is a complex phenomena significantly affected by the seam running across the middle of the membrane, discrete corner loads, and gravity. The pattern is marginally stable, changing somewhat with small external disturbances such as slight air currents. To obtain a consistent set of images, the four cameras were again fired simultaneously, freezing the shape. Using attached retro-reflective targets in this application is clearly inappropriate.

To increase contrast, a higher powered theater projector was used to create a grid of approximately 5000 dots on a $0.9 \times 0.5$ meter area on the right side of the sail, as indicated by the box in Figure 1. The same four consumer digital cameras used in the low resolution test, arranged in the same configuration as before, simultaneously photographed the grid. The reflectivity of the aluminized coating of the Kapton that previously led to the use of long exposure times was again a complicating factor, but was compensated for much more effectively. The higher powered projector and smaller distances involved allowed exposure times below 30 seconds, and the cameras were set to identical exposure values instead of the identical shutter speeds used previously. An exposure value, which indicates the over- or under-exposure of the image, of $-1.0 \mathrm{set}$ different shutter speeds in each camera ranging from 15 to 30 seconds, resulting in images of similar contrast.
Two of the images used in the processing are shown in Figure 6. The images are nearly optimal white-on-black images. The effect of ambient room lighting on the membrane has been eliminated and while a contrast gradient (inconsistent target contrast across the image) is still present, each image contains useful data out to the corners of the projected pattern. Note that while hot spots still caused a loss of data in the image on the right, the nearly uniform contrast in all of the images enabled redundancy that minimized the negative impact of the hot spots on the final outcome. In general the location of hot spots varies from one image to the next.

Figure 7 shows an image of the completed project created with the 3-D Viewer in the photogrammetry software. The graphic shows the camera locations at $90^{\circ}$ angles of separation, their relative orientations and fields of view, and the final point cloud, all appearing as expected. Figure 8 is a magnified view of the upper left corner of the grid visible in Figure 6, after the target marking was completed. Evident is a higher resolution compared with the previous application, indicating that the data points are sufficiently dense to measure the visible wrinkles. The greater density, however, does have a drawback; it is highly computationally intensive to calculate the positions of over 5000 points. The measurement in the low resolution case required hours to process, while this measurement required almost one week. Also apparent in Figure 8 is the sufficient contrast that allowed the computer to accurately mark the centers of almost all the targets using LSM centroiding. Automatic centroiding minimized the need for the human intervention necessary previously, thereby maximizing the accuracy of the marking process.

The average precision was calculated to be $0.062 \mathrm{~mm}$, yielding an overall precision on the area measured of 1:32,300. These numbers, combined with the high target density, allow for confidence in the measurement of wrinkles less than one millimeter in amplitude. The increase in precision from the low resolution test shows this measurement to be a marked improvement, demonstrating that high quality, highresolution measurements can be obtained for these structures using dot projection photogrammetry. Improvements in hardware and technique led to the gains, and further advances, detailed in the next section, will produce still better measurements.

The two views of the shaded surface shown in Figure 9 were created from the 3-D point cloud exported from the photogrammetry software. These maps, normally used for displaying topographic land features, clearly characterize the visible wrinkles in the Kapton membrane and the seam cutting horizontally across the center. The wrinkle amplitude can also be determined in Figure 9. The smallest wrinkle 
amplitude is approximately $0.25 \mathrm{~mm}$ and the average wrinkle amplitude is approximately $5.0 \mathrm{~mm}$. The seam and wrinkles visible in the shaded surface match the pattern seen in Figure 1. Note the amplitude in the Zdirection has been exaggerated for better viewing.

These results demonstrate that it is possible using careful experimental technique to make noncontact photogrammetric measurements of highly reflective membranes that accurately characterize their static shape, including wrinkling. These measurements are the first of sufficient quality to validate analytical models of this configuration. More powerful projectors will allow high-resolution measurements such as these on even large gossamer structures.

\section{High Density Full-field Two Meter Kapton Solar Sail}

The high density full-field test described here was designed to generate measurements of comparable resolution to the results in the above high resolution wrinkle test over the entire surface of the two meter Kapton solar sail instead of on only a small section. The same test article was used once again, however, the sail was rotated slightly, as shown in Figure 10, to create a small gravity-induced billow in the bottom half of the membrane. The billow will demonstrate the ability of the method to simultaneously measure both medium-amplitude wrinkles and global non-planar shapes. Four professional digital cameras at $90^{\circ}$ angles of separation at the corners of the membrane simultaneously photographed a grid of approximately 10,800 targets created by high power industrial flash projector. The use of the professional cameras and turn-key projector signify a transition to professional grade hardware designed to yield full-field, highresolution, high precision measurements the two meter solar sail structure.

One image used in the photogrammetric processing is shown in Figure 11. Ambient room lighting was minimized, but data was still lost due to the intensity of the flash projector. Because of the billow in the sail and the angles involved, a percentage of the light reflected by the membrane fell onto the floor. The high intensity of the projector meant a large amount of light, when scattered in all directions by the floor, imaged back onto the membrane. These images of the light on the floor were of sufficient intensity to wash out the original points. Curling of the membrane and the large distances involved again led to hot spots in the images; however all of the data loss was compensated for again by the redundancy of four cameras.

A three dimensional point cloud similar to those seen in Figures 2 and 7 again resulted from the photogrammetric processing. The time required to generate the final project, however, was much greater than in the two previous examples (three weeks vs. one). The current photogrammetry software was not designed to process such a large number of points, over 10,000 in this measurement, or to handle such close point spacing. The loss of data caused by hot spots and the image from the floor, shown in the top portion of Figure 12, added to the overall complexity of the processing, making this an extremely demanding and complicated measurement. Also evident in Figure 12 is the ability of the software to mark the projected dots using the LSM centroiding, as well as the true resolution of the projected pattern (impossible to see in Figure 11). In this case, the number of dots per meter is not quite as high as that used for the wrinkle application above, but it is the highest currently achievable with the available hardware. Approximately 10,800 dots were projected onto the membrane, and with the small size of the targets and the distance from the test article to the cameras (approximately three meters), some dots appeared as small as five pixels in diameter with as little as five pixels spacing. Higher pattern resolution or larger dots are therefore impractical for this measurement given the current hardware configuration. The most important improvement of this measurement over the previous one is evident in the precision numbers. Over the four square meters of membrane area imaged, the professional system was precise to 59 microns versus 62 microns over less than 0.5 square meters imaged previously, meaning that the overall precision increased from 1:32,300 to $1: 50,800$.

The shaded surface shown in Figure 13 clearly characterizes all the medium-amplitude wrinkles, the seam, and the billow seen in Figure 10. This plot details the entire surface of the test article instead of just a small area as in the previous application. The wrinkles are clearly evident, but in addition it is possible to see the true size of the billow compared to the wrinkles. The measured wrinkles are on average five millimeters in amplitude, matching the measurement in the previous section, while the billow is almost ten times that size. Also apparent in Figure 13 is the tendency of the membrane to curl at the edges.

The shape characterization produced here is unique. A realistic solar sail model has never before been achieved in a full-field, totally non-contact manner to this resolution. This surface model is of sufficient quality to be used for validation of analytical models and for supporting development of hardware and software tools for future measurements and use in space, achieving both of the stated goals.

\section{Dynamic Analysis of a Two Meter Vellum Solar Sail}

Structural dynamic characterization is also important for the development and validation of analytical models supporting future designs of gossamer structures in general and solar sails in particular. In the dynamic analysis of these structures, 
as in the static characterization, non-contact measurement is necessary to avoid altering the responses of the objects being measured. Videogrammetry expands the methods and techniques of photogrammetry to multiple time steps yielding dynamic data. While other non-contact dynamic measurement techniques exist, they are not full-field. It was hoped that the dynamic data generated would be useful in simply tracking the overall shape of the membrane as a function of time. The accuracy of the measurement of the resonant frequencies and operating deflection shapes that were extracted, however, surpassed all expectations. [11]

Figure 14 shows the two meter solar sail test article measured in this application. The test article membranes are 100 micron thick Vellum, a diffuse white material ideal for dot projection since it scatters light almost equally in all directions regardless of the angle of incidence. This property enables the Vellum to yield a grid of uniform contrast from any camera angle. The test article is not designed to approximate actual solar sail material as is the test article used in the first three applications, but is designed as a research tool for measurement method development. Figure 15 shows the test setup used in the experiment. A grid of 49 dots was created on the right quadrant of the four quadrant solar sail by a digital projector and retro-reflective targets were attached to the booms. A long-stroke electrodynamic shaker attached to the tip of the lower right boom excited the structure with a pseudo-random forcing function. A turn-key scanning laser vibrometer measured the frequency response function of the sail quadrant and its corresponding operating deflection shapes, and is used as a standard against which to compare and determine the validity of the videogrammetry results.

At high resolutions, the vibrometer measures thousands of FFT lines at each point, 1600 in this application, to produce detailed results. However this form of data acquisition by scanning each point individually is slow at low frequencies, requiring several hours to run one test. Videogrammetry is attractive because of its ability to measure all of the points simultaneously. For the results presented below, the cameras recorded data for approximately ten seconds gathering a total of only 384 frames each instead of several sets of 3200 samples (twice the number of FFT lines) taken by the vibrometer at each point. While the videogrammetric results shown below are arguably not as clean as the vibrometer data, they are full-field, simultaneous measurements taken in just a few seconds instead of hours.

The position versus time videogrammetry data was loaded into modal analysis software for interpretation where two different computational methods were used. The first involved overlaying the time histories of individual points and calculating the
Fourier transform of the joint history. The software then animated the Operating Deflection Shapes (ODS) at each peak in the frequency domain. The second method of analysis calculated frequency response functions (FRF). Here only one degree of freedom was used, the out-of-plane displacement in the $\mathrm{Z}$ direction, identical to that used by the vibrometer. For the videogrammetry experiment the input signal was not measured, so a non-node reference point on the membrane was specified enabling the software to calculate the FRF's and animate shapes again at the peaks in the frequency domain. The combined results of both of these methods of analysis are compared against the results of the laser vibrometer measurements in Table 1 below. While neither the ODS or FRF method is currently considered to be entirely accurate, some combination of the two will likely capture all dynamics below $5 \mathrm{~Hz}$. Note that the non-linear nature of the deflection shapes, their coupling, and the inability to compensate for atmospheric damping mean that the shapes shown in Figures 16 through 20 are not actually mode shapes, and that the frequencies listed in Table 1 are not actually modal frequencies. These can alternatively be referred to as "structural resonances" and "resonant frequencies." [12, 13]

Table 1 - Identified resonant frequencies of the $2 \mathrm{~m}$ Vellum solar sail

\begin{tabular}{|c|c|}
\hline Laser Vibrometer & Videogrammetry \\
\hline $1.75 \mathrm{~Hz}$ & $1.77 \mathrm{~Hz}(\mathrm{ODS})$ \\
\hline \multirow{2}{*}{$2.65 \mathrm{~Hz}$} & $2.49 \mathrm{~Hz}(\mathrm{FRF}$, ODS $)$ \\
\cline { 2 - 2 } & $2.81 \mathrm{~Hz}(\mathrm{ODS})$ \\
\hline $3.34 \mathrm{~Hz}$ & $3.28 \mathrm{~Hz}(\mathrm{FRF})$ \\
\hline $3.67 \mathrm{~Hz}$ & $3.69 \mathrm{~Hz}(\mathrm{ODS}, \mathrm{FRF})$ \\
\hline $4.74 \mathrm{~Hz}$ & $4.75 \mathrm{~Hz}(\mathrm{ODS}, \mathrm{FRF})$ \\
\hline
\end{tabular}

Examination of Table 1 reveals good correlation between the results found using laser vibrometry and videogrammetry. While several inconsistencies are evident, overall the resonant frequencies were measured to within $0.2 \mathrm{~Hz}$ of each other. The deflection shapes are shown in Figures 16 through 20.

The first two shapes shown in Figures 16 and 17 are basically boom driven modes. The first deflection shape shows that the booms are in phase, and the second shows the booms to be out of phase with the membrane following in a first order fashion. Figure 18 shows the third deflection shape of the system, with the booms in phase and the membrane in a second order configuration. The fourth deflection shape (Figure 19) shows the booms to be in phase with a large amplitude first order billow of the membrane. Finally, the fifth deflection shape in Figure 20 shows a saddle configuration for the membrane. In all of the figures videogrammetry produced similar shapes at similar frequencies as the vibrometer, demonstrating the

$-5-$ 
potential of the full-field non-contact dynamic characterization technique.

Videogrammetry can only measure frequencies up to approximately one half the frame rate of the cameras without using stroboscopic flashes. This limitation will not generally be a hindrance for use on gossamer structures because the dominant modes will occur below $5.0 \mathrm{~Hz}$. Also, the displacement of the test article must be large enough to be detected by the cameras, whereas the vibrometer is much more sensitive. The above results show videogrammetry to be capable of achieving full-field non-contact dynamic measurements of solar sail structures.

\section{Conclusions}

Accurate static and dynamic characterization of solar sail test articles is vital for creating and validating high-fidelity analytical models, developing space quality hardware, and future advancement of the technology. Obtaining accurate static and dynamic measurements of the highly reflective ultra-thin membranes, however, is not a trivial task. Dot projection photogrammetry and videogrammetry were used to avoid physically attaching any devices or targets to the membranes that might alter their static and dynamic properties. The described tests show a progression from consumer hardware that produced low resolution measurements to professional hardware that produced high resolution, full-field measurements of local wrinkles and global deformations. Dynamic measurements were performed using an adaptation of photogrammetry to time-dependant data called videogrammetry. The data yielded resonance frequencies and deflection shapes which were compared to data from a scanning laser vibrometer. The photogrammetry and videogrammetry methods generated results of sufficient quality to be used in the validation of numerical models as well as future development of solar sails.

\section{$\underline{\text { Acknowledgements }}$}

The authors would like to thank Dr. Keith Belvin, Dr. Kara Slade, Mr. Tom Jones, Dr. Adrian Dorrington, Dr. Paul Danehy, and all the members of the Ultra-lightweight and Inflatable Structures group at NASA Langley Research Center (LaRC), the members of the Structural Dynamics Branch at LaRC, Dr. Jack Leifer of the University of Kentucky, Dr. Joe Blandino of James Madison University, and Dr. Paul Cooper, Dr. Robert Blanchard, and Dr. Robert Sandusky of George Washington University. This work was performed in partial satisfaction of the Master of Science degree under a Graduate Research Scholar Assistantship provided by NASA Langley through the George Washington University.

\section{References}

[1] Jenkins, C. H. M. (editor), Gossamer Spacecraft: Membrane and Inflatable Structures Technology for Space Applications, Vol. 191, Progress in Astronautics and Aeronautics, AIAA, Reston, VA, 2001.

[2] Chmielewski, A. B., Moore, C., and Howard, R., "The Gossamer Initiative," IEEE 0-7803-5846-5/00, 2000.

[3] McInnes, C. R., Solar Sailing - Technology, Dynamics and Mission Applications, Praxis Publishing Ltd, Chichester, UK, 1999.

[4] West, J. L., and Derbes, B., "Solar Sail Vehicle System Design for the GEOStorm Warning Mission," AIAA 20005326, 2000.

[5] McInnes, C. R., "Solar Sail Mission Opportunities," Royal Astronomical Society Discussion Meeting, 10 May 2002.

[6] Spieth, D. and Zubrin, R., "Ultra-Thin Solar Sails for Interstellar Travel - Phase I Final Report," NASA Institute for Advanced Concepts, Pioneer Astronautics Inc., December 1999.

[7] Pappa, R. S., Jones, T. W., Black, J. T., Walford, A., Robson, S., and Shortis, M. R., "Photogrammetry Methodology Development for Gossamer Space Structures," Sound and Vibration, Vol. 36, No. 8, August 2002, pp. 12-21.

[8] Mikhail, E. M., Bethel, J. S., and McGlone, J. C., Introduction to Modern Photogrammetry, John Wiley \& Sons, New York, NY, 2001.

[9] Eos Systems, Inc., PhotoModeler Pro User's Manual, Version 5, Vancouver, B.C., Canada, 2003.

[10] Pappa, R. S., Black, J. T., Blandino, J. R., Jones, T. W., Danehy, P. M., and Dorrington, A. A., "Dot Projection Photogrammetry and Videogrammetry of Gossamer Space Structures," to appear in the Journal of Spacecraft and Rockets, NASA/TM-2003-212146, February 2003.

[11] Black, J. T. and Pappa, R. S., "Videogrammetry Using Projected Circular Targets: Proof-of-Concept Test," NASA/TM-2003-212148, February 2003.

[12] Blandino, J. R., Pappa, R. S., and Black J. T., "Modal Identification of Membrane Structures with Videogrammetry and Laser Vibrometry," AIAA Paper 2003-1745, April 2003.

[13] Batel, M., "Operational Modal Analysis - Another Way of Doing Modal Testing," Sound and Vibration, Vol. 36, No. 8, August 2002, pp. 22-27. 


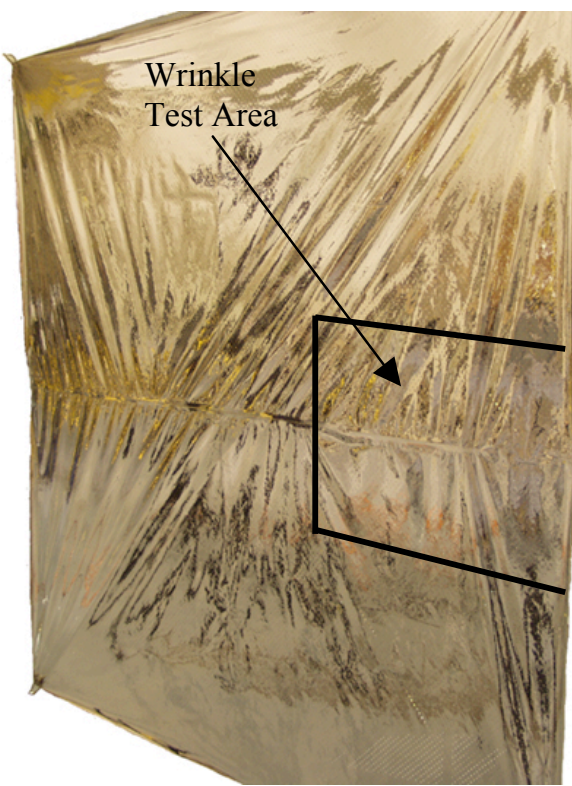

Figure 1 - Two meter Kapton single quadrant solar sail

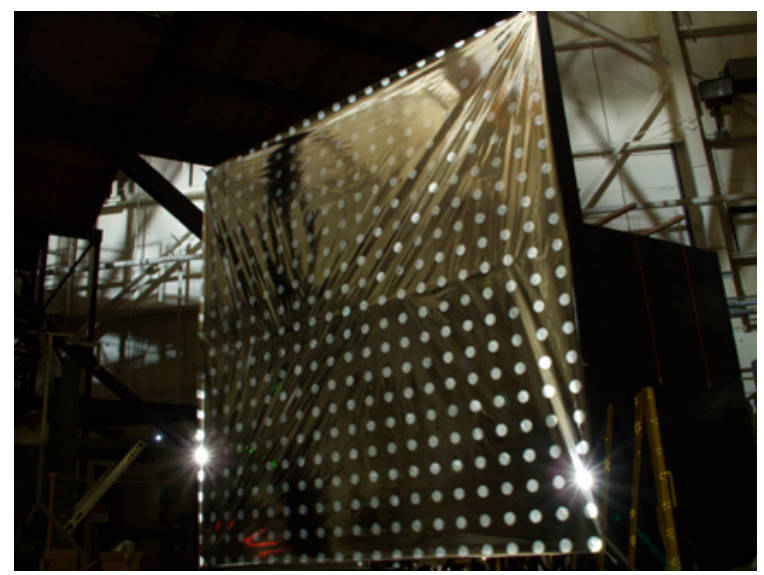

Figure 3 - Image used in photogrammetric processing (1 of 4 )

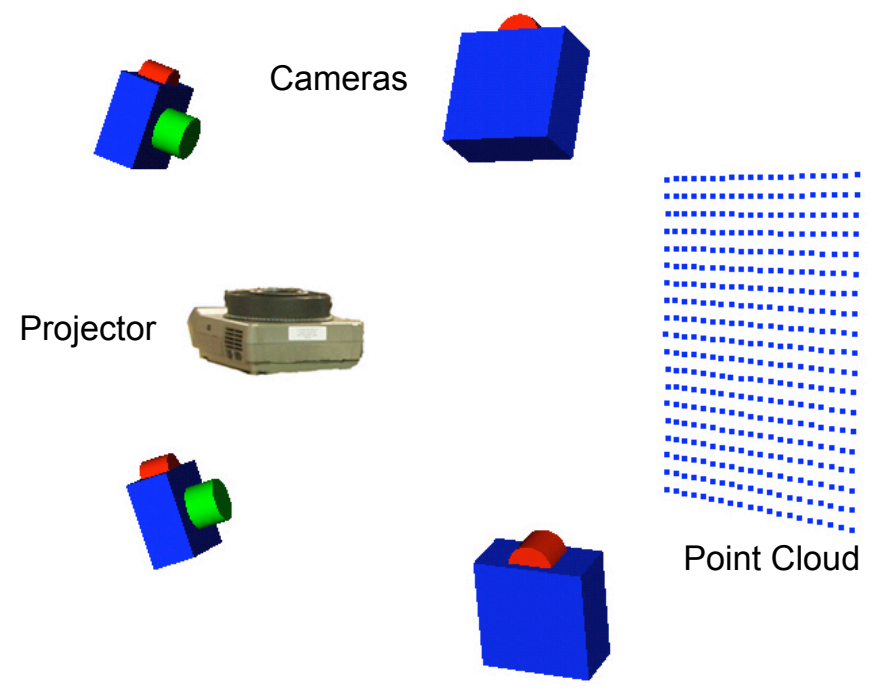

Figure 2 - Kapton two meter full field test setup

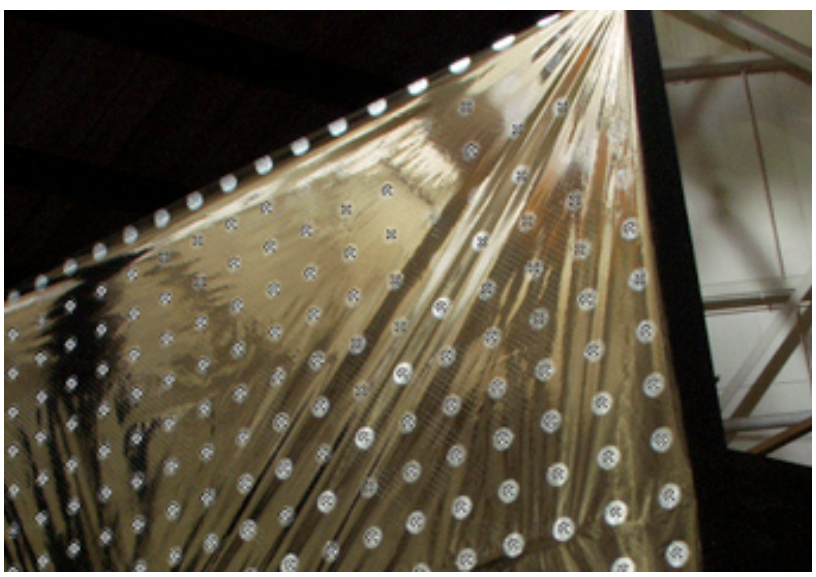

Figure 4 - Marked points in upper right corner of the solar sail test article

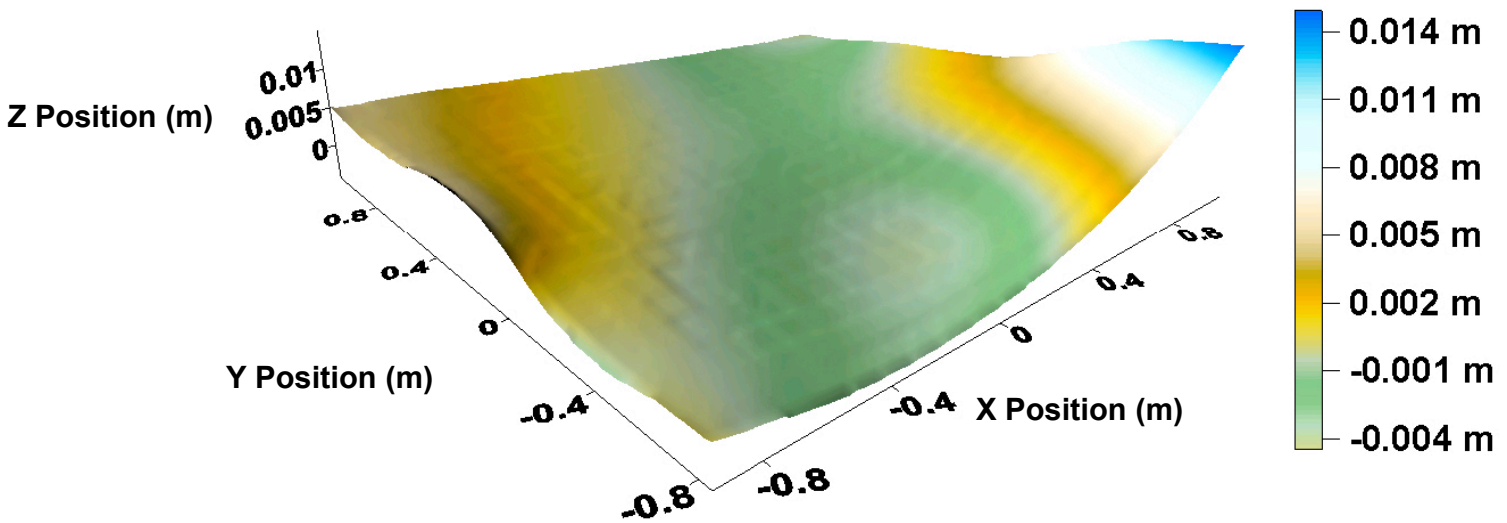

Figure 5 - Shaded surface for low density measurements of the solar sail test article (Z position amplified) 

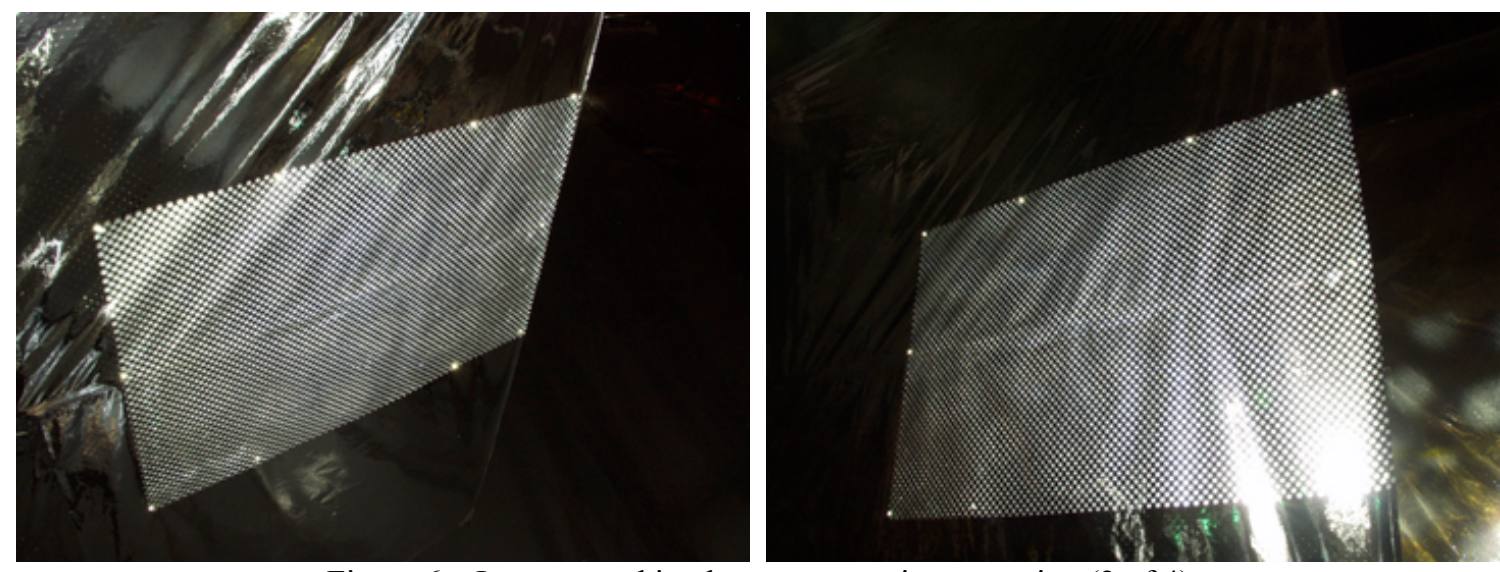

Figure 6 - Images used in photogrammetric processing (2 of 4)

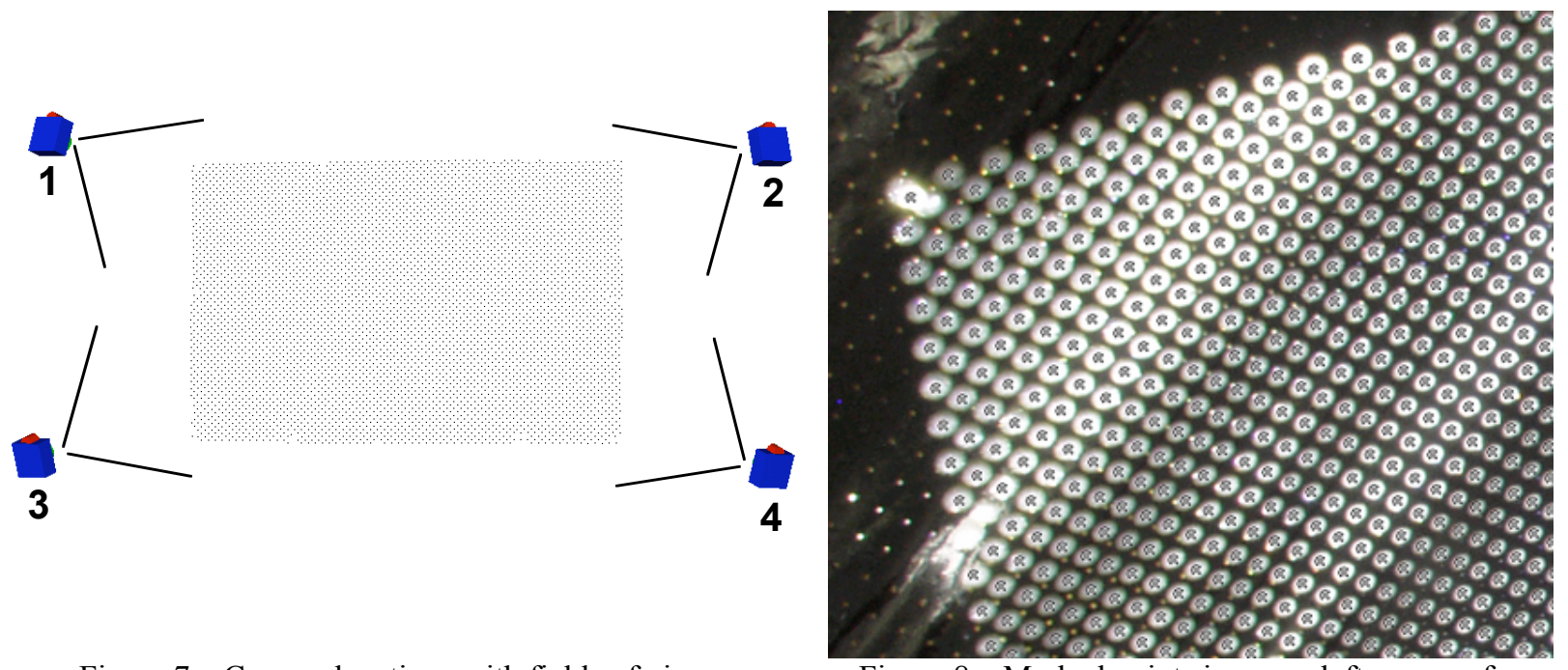

Figure 7 - Camera locations with fields of view

Figure 8 - Marked points in upper left corner of the area imaged
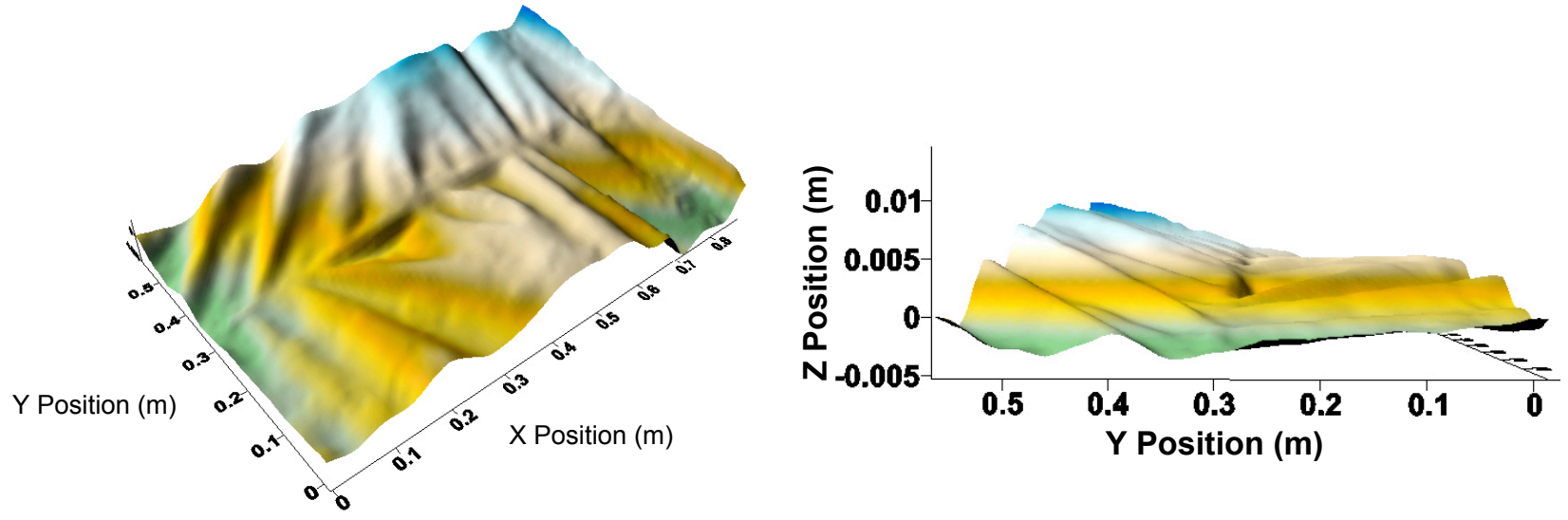

Figure 9 - Two views of contour surface 


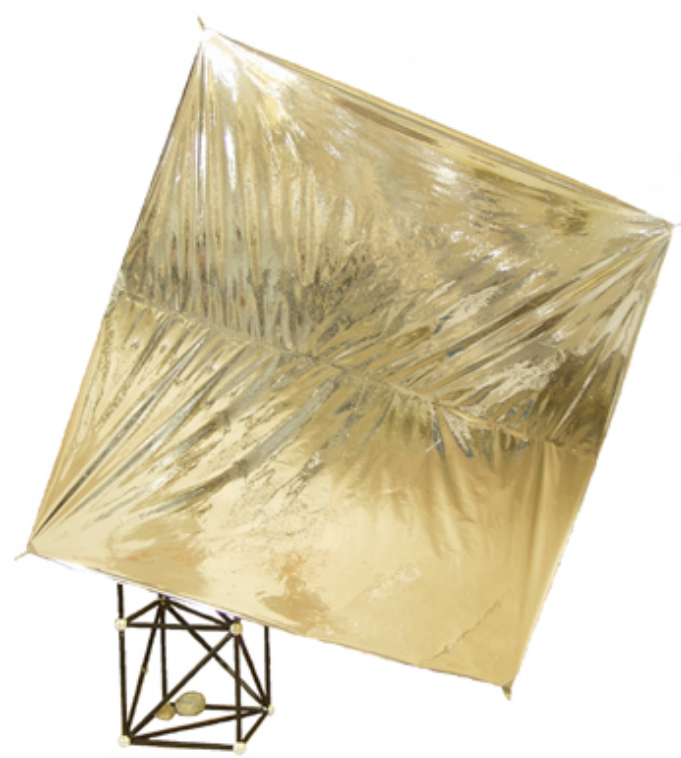

Figure 10 - Two meter Kapton solar sail test article rotated slightly with gravity-induced billow

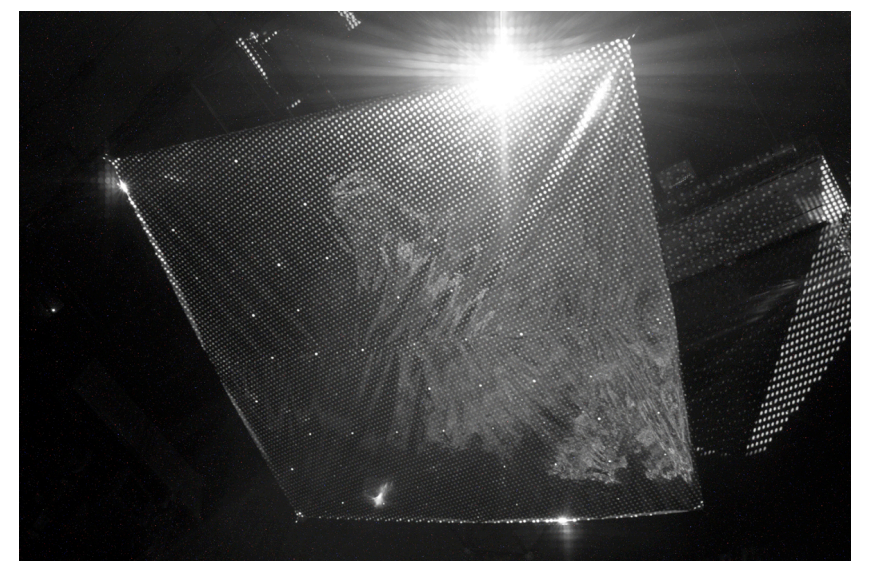

Figure 11 - Image used in photogrammetric processing ( 1 of 4$)$

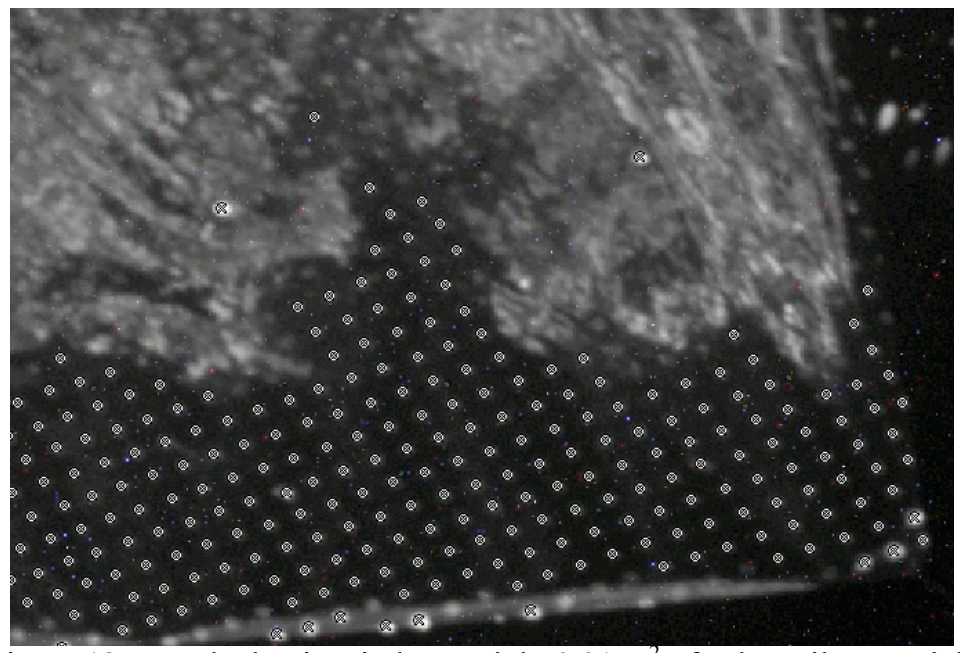

Figure 12 - Marked points in lower right $0.01 \mathrm{~m}^{2}$ of solar sail test article

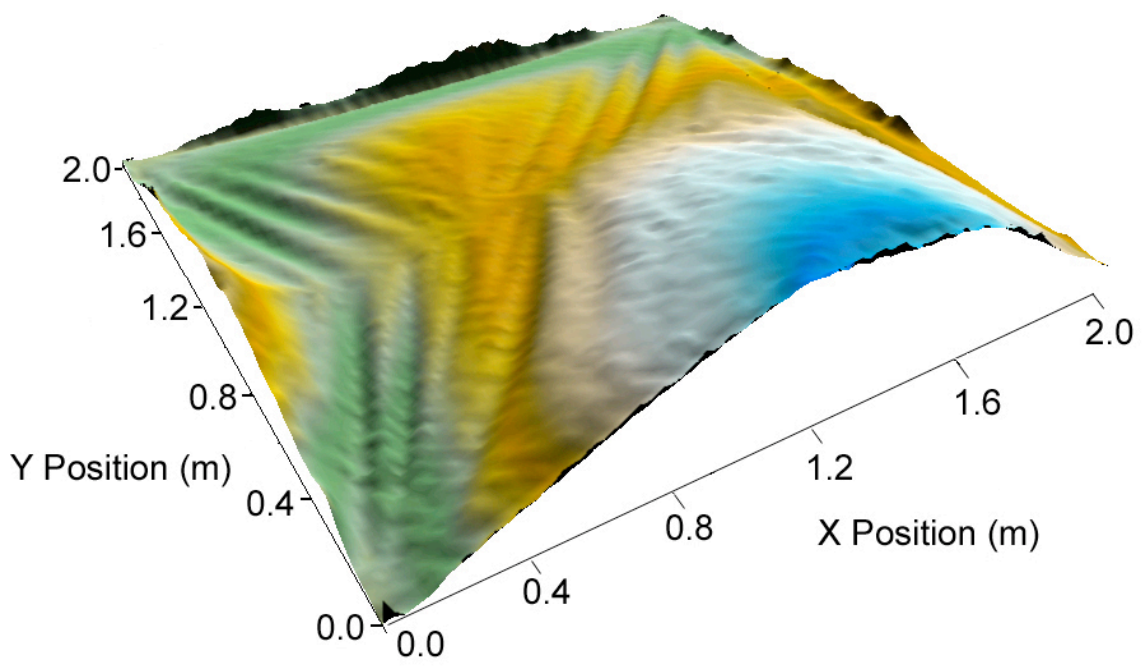

$0.06 \mathrm{~m}$

$0.05 \mathrm{~m}$

$0.04 \mathrm{~m}$

$-0.03 \mathrm{~m}$

$0.02 \mathrm{~m}$

$0.01 \mathrm{~m}$

$\mathbf{0} \mathbf{m}$

Figure 13 - Two views of the contour surface generated 


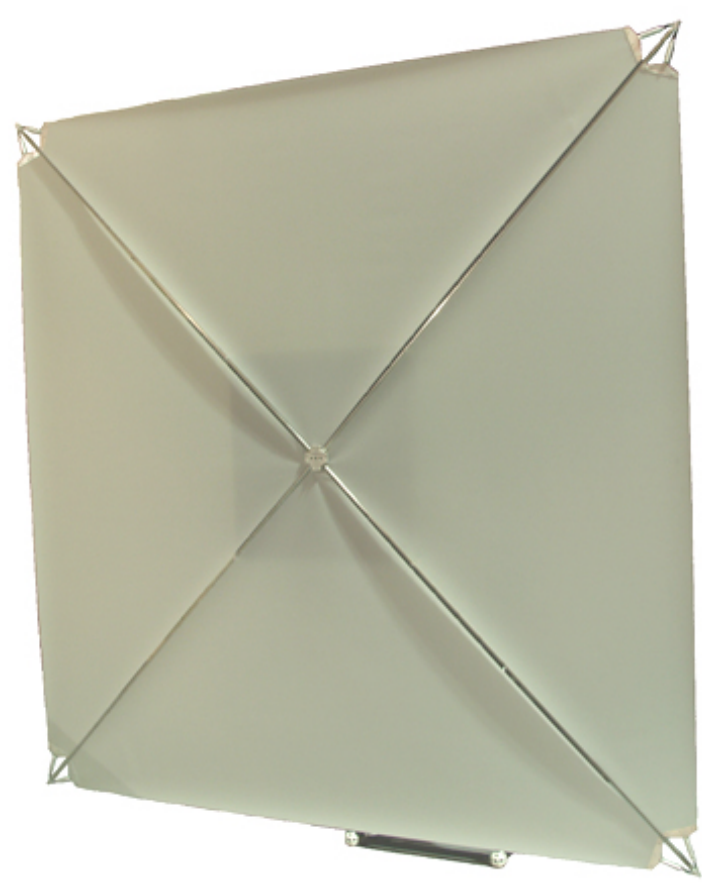

Figure 14 - Two meter four quadrant Vellum solar sail test article

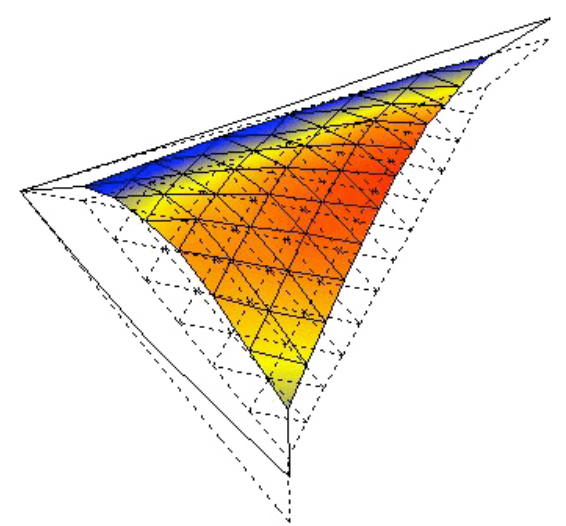

(a) $1.75 \mathrm{~Hz}$ Laser Vibrometer

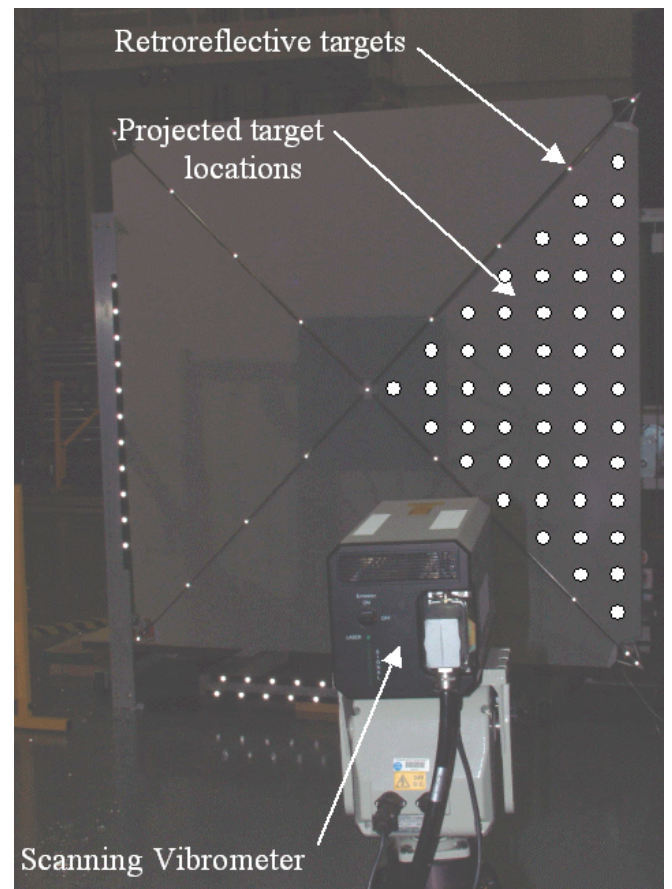

Figure 15 - Test setup for dynamic characterization

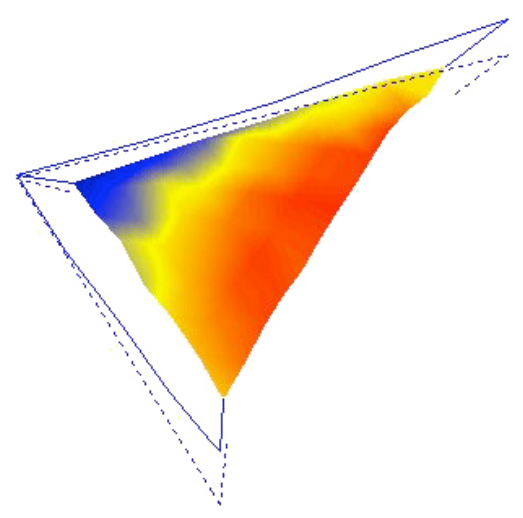

(b) $1.77 \mathrm{~Hz}$ Videogrammetry (ODS)

Figure 16 - First deflection shape comparison for Vellum solar sail test article

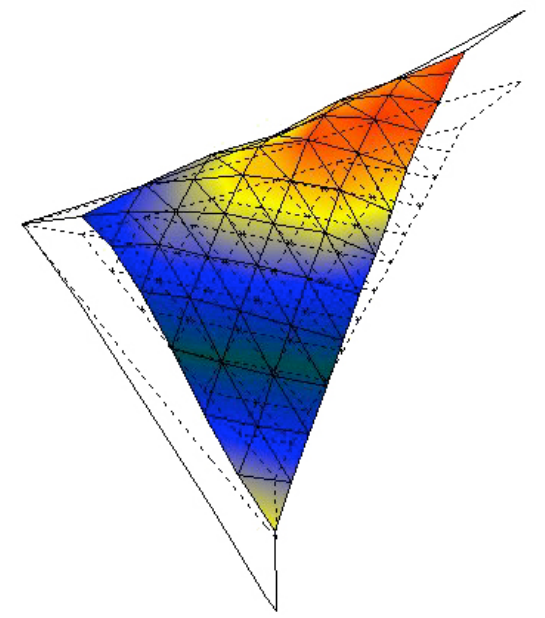

(a) $2.65 \mathrm{~Hz}$ Laser Vibrometer

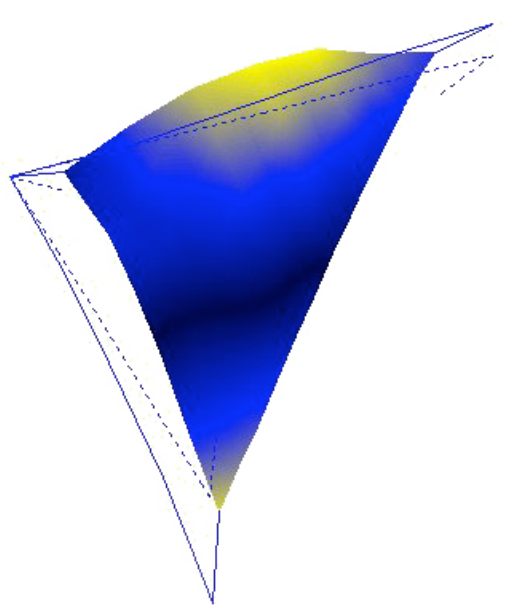

(b) $2.49 \mathrm{~Hz}$ Videogrammetry (FRF)

Figure 17 - Second deflection shape comparison for Vellum solar sail test article 


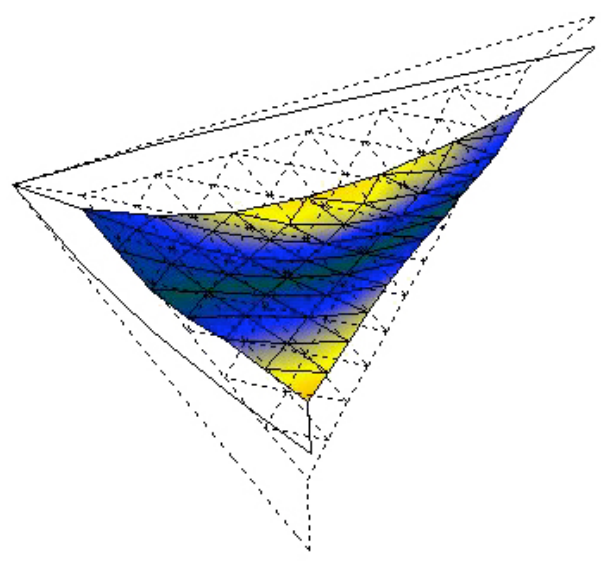

(a) $3.34 \mathrm{~Hz}$ Laser Vibrometer

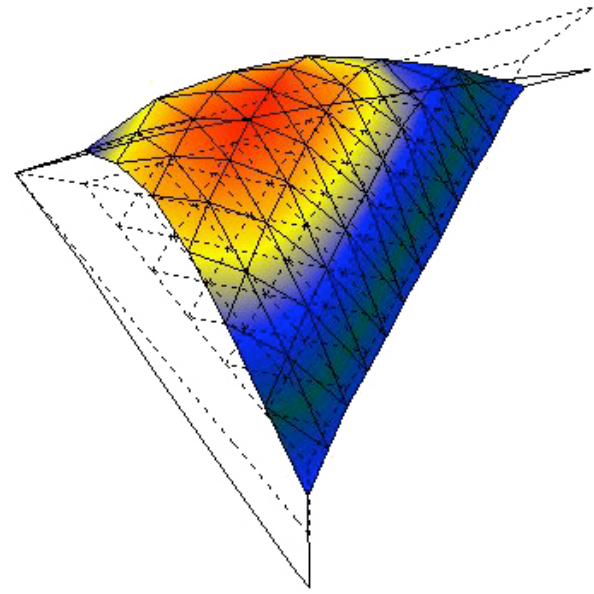

(a) $3.67 \mathrm{~Hz}$ Laser Vibrometer

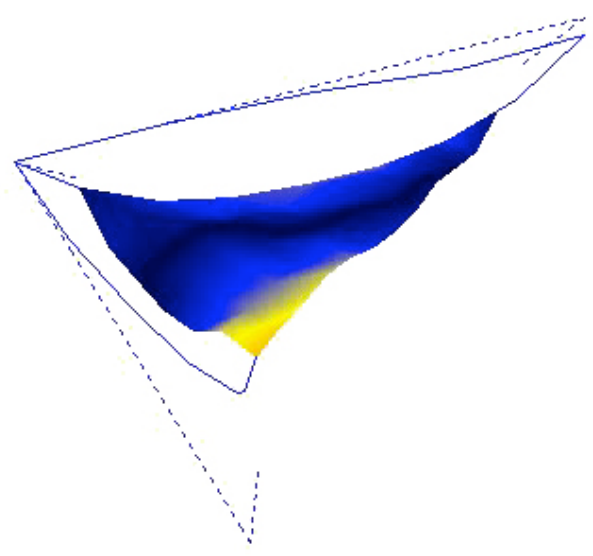

(b) $3.28 \mathrm{~Hz}$ Videogrammetry (FRF)

Figure 18 - Third deflection shape comparison for Vellum solar sail test article

Figure 19 - Fourth deflection shape comparison for Vellum solar sail test article

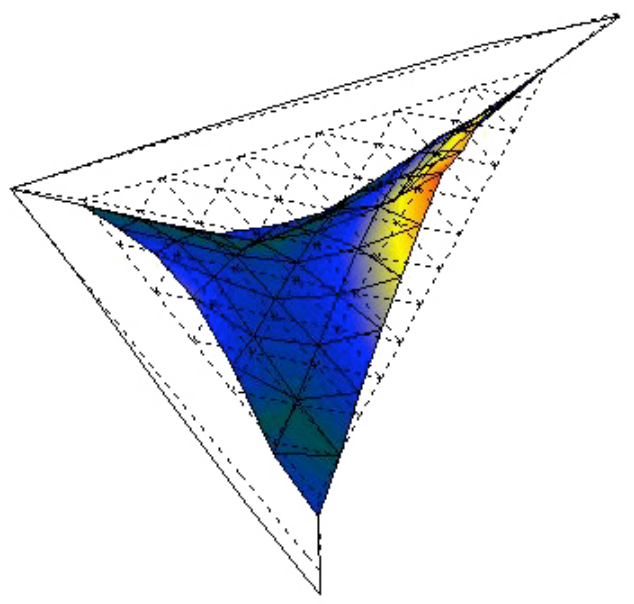

(a) $4.74 \mathrm{~Hz}$ Laser Vibrometer

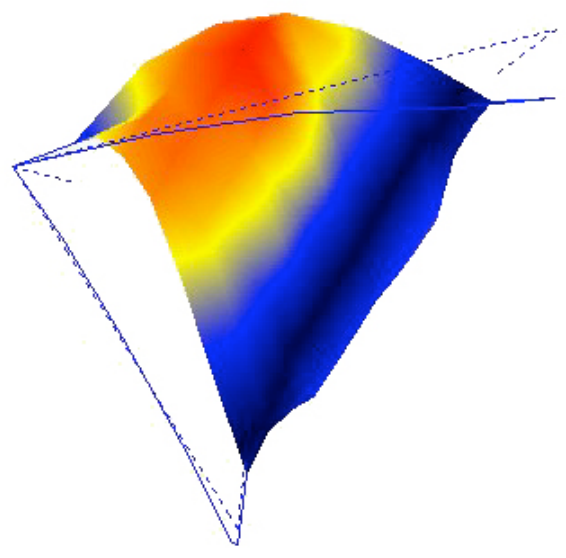

(b) $3.69 \mathrm{~Hz}$ Videogrammetry (FRF)

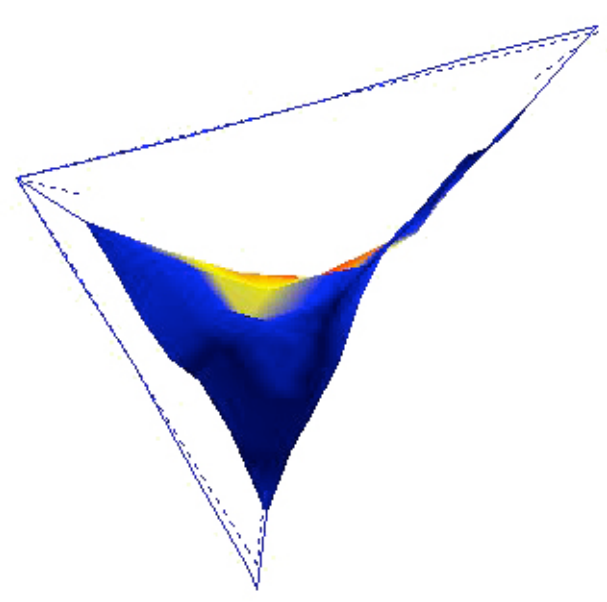

(b) $4.75 \mathrm{~Hz}$ Videogrammetry (FRF)

Figure 20 - Fifth deflection shape comparison for Vellum solar sail test article 